\title{
Newborn screening and carrier screening for cystic fibrosis: alternative or complementary?
}

\author{
Carlo Castellani ${ }^{1}$ and John Massie ${ }^{2,3,4}$
}

Affiliations: ${ }^{1}$ Cystic Fibrosis Centre, Azienda Ospedaliera Universitaria Integrata, Verona, Italy. ${ }^{2}$ Dept of Respiratory Medicine, Royal Children's Hospital, Melbourne, ${ }^{3}$ Murdoch Childrens Research Institute, Melbourne, and ${ }^{4}$ Dept of Paediatrics, University of Melbourne, Melbourne, Australia.

Correspondence: C. Castellani, Cystic Fibrosis Center, Azienda Ospedaliera Universitaria Integrata, Piazzale Stefani 1, 37126 Verona, Italy. E-mail: carlo.castellanidospedaleuniverona.it

0

@ERSpublications

CF newborn and carrier screening are interconnected but presently neither can replace the other http://ow.ly/qhDFD

Cystic fibrosis (CF) is the most frequent, life limiting, autosomal recessive diseases in Caucasians. It is caused by mutations in the CF transmembrane conductance regulator (CFTR) gene. Approximately 2000 mutations have been identified, of which $\sim 120$ are responsible for the vast majority of disease cases [1].

The last decade has seen the increasing application of two models of screening related to CF, newborn screening and community-wide carrier screening (carrier screening) [2, 3]. Newborn screening for CF has now been implemented in the majority of countries where CF is common, including North America, Australia and several parts of Europe [3]. Virtually all babies born in these places will have been screened and those diagnosed with CF will have access to early treatment. Carrier screening is less extensively used, but has been growing in acceptance, and it is offered to females or couples in the USA and in parts of Europe and Australia $[2,4]$. In the USA carrier screening has been recommended by the American College of Obstetricians and Gynaecologists and by the American College of Medical Genetics [5, 6], and, although there is not an established public health programme, millions of carrier tests have been performed [7].

Newborn and carrier screening for CF have different purposes, but also common features that may create some confusion among health practitioners as to their respective characteristics and goals.

CF newborn screening is a complex procedure that uses multiple step combinations of tests on dried blood spots. The first tier is always a measurement of immunoreactive trypsinogen (IRT), followed in IRT-positive babies by other tests, which usually include mutation analysis of the CFTR gene. The aim is to identify neonates at high risk of having CF, these infants are then referred to a diagnostic service to confirm (true positives) or refute (false positives) the diagnosis. In several countries CF has been added to existing newborn screening programmes and coverage of the screened population is nearly $100 \%$. Specific details on CF newborn screening are usually embedded in the general newborn screening information material for parents, and even with the current move to formalise consent, refusal is very rare.

In contrast, the aim of carrier screening is to detect adult heterozygotes and, thereby, enable informed reproductive choices before the birth of the first child with CF. CFTR mutation analysis is offered to individuals without a family history of $\mathrm{CF}$, which is a population with no a priori increased risk for having a child with CF [2]. Most, but not all, heterozygotes can be identified by the current molecular analysis techniques. Carrier screening is not routine in any country and is restricted by law in some states. Its application has been limited by various ethical questions. These include: the potential for misunderstanding

Received: July 232013 | Accepted after revision: Sept 302013

Conflict of interest: None declared.

Copyright @ERS 2014 
the residual carrier risk after a negative test; issues of equitable allocation; and improving outlook for patients with CF. Carrier screening requires pretest information, along with the availability of genetic counselling if a carrier couple is detected. The uptake is variable and affected by personal (stage of life, awareness of CF, awareness of screening availability, perceived vulnerability to being a carrier, cost and attitudes to reproductive options) and physician factors (knowledge of CF, awareness of screening availability and time constraints) [2, 8, 9].The workflows of newborn and carrier screenings are illustrated in figure 1.

Although these two screening approaches are different, with respect to aims, availability and target population, CFTR mutation analysis has a major role in both, being the core of any carrier screening strategy, and a valuable second tier test in most newborn screening protocols. In carrier screening the appropriate choice of mutations is the major determinant of programme sensitivity. In newborn screenings the main purpose of mutation analysis is to improve screening specificity by limiting the number of IRTpositive newborns recalled for further investigation.

\section{Effects of carrier screening on newborn screening}

Carrier screening detects couples who for each pregnancy have a $25 \%$ probability of having a child with CF. These couples can choose from various reproductive alternatives, which include the full range of options if screening occurs before conception, and more limited choices if a pregnancy has already started. Several of these options, i.e. change of partner, pre-implantation or prenatal diagnosis, affected foetus termination and adoption will lower the live birth prevalence of CF, as has been reported in the UK [10], USA [11], and Italy [4].

The decrease in the numbers of neonates with CF would not be associated with an equivalent decrease of false positives, with the final effect of lowering the positive predictive value (ratio between true positives and the total of true positives and false positives) of the newborn screening system.

Furthermore, carrier screening would not reduce the number of infants in which CF can neither be confirmed nor excluded after newborn screening [12]. These equivocal situations are considered by most an unwanted side-effect of newborn screening. Over time a few of these infants will develop CF but many others will remain healthy. It is unlikely that carrier screening will reduce the incidence of this phenomenon, because gene sequence variants associated with it are usually not included in standard screening mutation panels. Moreover, it has been anticipated that the potentially milder or absent clinical picture should have no consequence on parents' reproductive plans. Thus, the ratio between CF and these inconclusive diagnoses, which in parts of the USA has been reported to be close to 2:1 [13], may be expected to decrease and the number of equivocal conditions detected by newborn screening to approximate that of new CF cases.

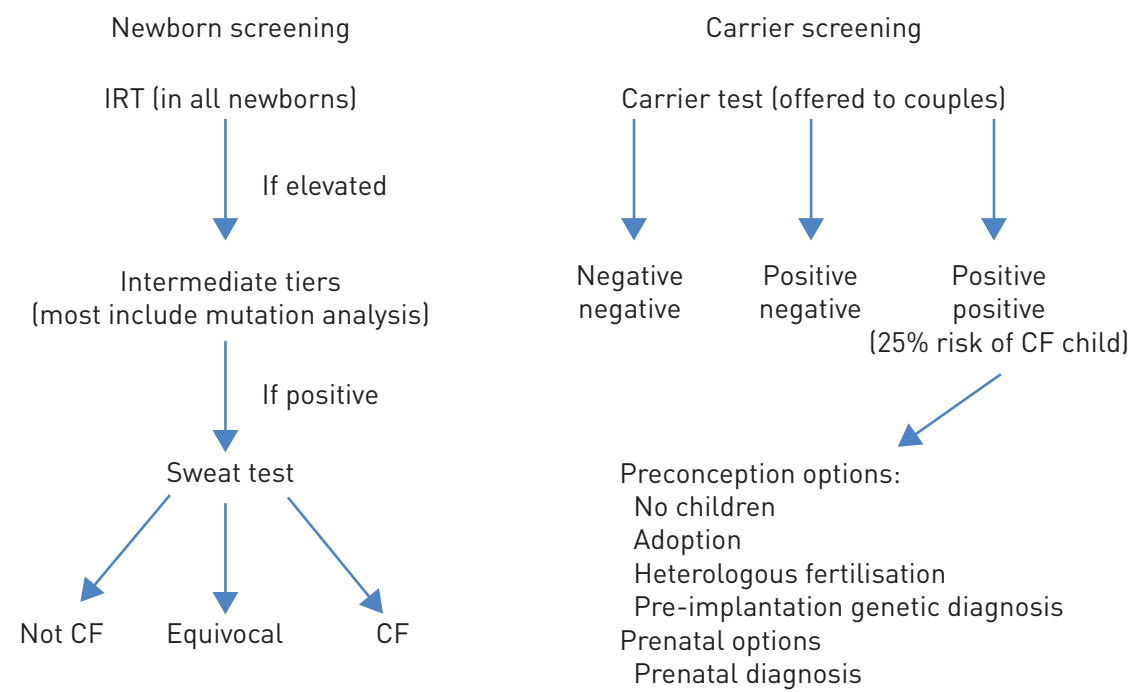

FIGURE 1 Workflow of newborn and carrier screening for cystic fibrosis (CF). Algorithms may vary according to local strategic choices. The carrier test can be offered to one partner within a couple and only if positive then to the other, or simultaneously to both partners. IRT: immunoreactive trypsinogen. 


\section{Effects of newborn screening on carrier screening}

Families of affected infants diagnosed through newborn screening are offered genetic counselling. Mutation analysis in relatives has a higher yield than carrier screening in the general population, both because of the higher a priori carrier risk and of the greater awareness of the significance of the test in families with a personal experience of CF. Even so, most babies with CF are born to families with no history of CF, and family testing following newborn screening is expected to have modest effects on CF incidence [14].

Depending on the configuration of the newborn screening protocol, a variable number of false positives are detected, who usually turn out to be carriers. Although heterozygotes tend to have higher IRT levels than non-heterozygotes [15], the number of carriers detected by newborn screening is small compared with the entire neonatal heterozygous population, as only the blood spots from the relatively few babies with an elevated IRT proceed to mutation analysis. Families where a carrier is found are also offered genetic counselling, but even after expanding the test to relatives, the vast majority of carriers are not detected by newborn screening [16].

\section{Costs effectiveness}

One of the advantages of the introduction of CF newborn screening is that it has been added to existing newborn screening programmes, which significantly defrays the cost. The IRT assay is relatively cheap and the more expensive CFTR mutation analysis is performed only in the small subset of IRT-positive newborns. The cost per infant diagnosed can be calculated, and it can even be estimated that savings are possible by getting infants into care early and delaying the onset of expensive medical requirements for advanced CF disease [17]. Conversely, the introduction of carrier screening for CF involves the establishment of a service as there is very little in the way of inherited disease screening for couples planning a pregnancy or for pregnant females in most countries. In contrast to newborn screening, established carrier screening programmes will result in a lower live birth prevalence of CF and the potential for an alternative source of cost savings. The cost effectiveness data, with regard to carrier screening, suffer from methodological variations in terms of expenditure for care and difficulties in estimating what the uptake would be for this service. However, two recent studies in Australia [18, 19], using different estimates of the cost of care, indicate that, after the initial investment to establish a carrier screening programme, cost savings are possible.

There are no studies to calculate the direct relationship between CF carrier and newborn screening. It is plausible that the decrease in incidence following carrier screening implementation would have no impact on the total cost of newborn screening programmes, but the cost per infant detected would increase.

\section{Could carrier screening replace newborn screening?}

Depending on their attitudes and on the preconception or prenatal timing of the test, some carrier couples will elect to have no children with CF and others will pursue their original reproductive plans. The latter scenario would result in prompt CF diagnoses, either at birth, if prenatal diagnosis had been performed, or shortly afterwards by testing the newborn for the parental mutations and/or by carrying out an early sweat test. Thus, it is theoretically possible for carrier screening to replace newborn screening. However, a number of practical issues make it quite unlikely to happen.

First, newborn screening coverage rates include nearly all the neonatal population, while even the best planned community-wide carrier screening programmes do not reach the entire target group. An exception to this occurs in premarital programmes in some motivated communities, such as Ashkenazi Jews, a paradigm difficult to adopt in the general cultural framework [20].

Secondly, the sensitivity of CF newborn screening programmes is usually $>95 \%$, whereas in many carrier screening programmes is close to $85 \%$. Since it is necessary to identify both partners as carriers to offer a meaningful risk assessment of having an affected pregnancy, an $85 \%$ detection rate would identify only approximately three quarters of the carrier couples tested.

Finally, the guidelines recommend only testing for CF-causing mutations found in at least $0.5-1 \%$ of the local CF population [2]. This can further decrease the carrier test sensitivity in regions where the distribution of mutations is more heterogeneous, thus making the number of undetected heterozygotes and the residual carrier risk too high to justify the implementation of carrier screening.

While carrier screening is unlikely to replace newborn screening, there are some potential advantages to the infant diagnosed in utero. There is growing evidence that CF lung damage starts very early in life, and may even be present at the time of diagnosis by newborn screening at 4-6 weeks of age [21]. For 5\% of the CF population (those with the p.Gly551Asp mutation) there is a newly developed medication (ivacaftor) that improves chloride transport through CFTR [22]. Further compounds aimed at other mutations are under development. 
Although ivacaftor is currently licensed to be used from 6 years of age, it is reasonable to suppose that the earliest possible initiation of therapy would provide the greatest benefits. A phase III clinical trial on the same compound in children aged 2-5 years is currently recruiting patients [23]. Furthermore, 20-30\% of infants with CF have residual exocrine pancreatic function at birth that is then lost over 6-12 months. Early therapy with CFTR function restoring medications might prevent such loss and obviate the need for replacement enzymes. Taking this argument one step further, treatment of pregnant females with an affected foetus may possibly prevent some of the in utero development of CF complications, such as meconium ileus, absent vas deferens and pancreatic exocrine insufficiency.

\section{Conclusion}

CF newborn and carrier screening have complementary roles and neither can replace the other. In contrast to newborn screening, carrier screening allows informed reproductive choices before the birth of a child with CF. Carrier screening may get infants into care earlier than newborn screening, but would miss more affected babies.

\section{References}

1 Sosnay PR, Siklosi KR, Van Goor F, et al. Defining the disease liability of variants in the cystic fibrosis transmembrane conductance regulator gene. Nat Genet 2013; 45: 1160-1167.

2 Castellani C, Macek M Jr, Cassiman JJ, et al. Benchmarks for cystic fibrosis carrier screening: a European consensus document. J Cyst Fibros 2010; 9: 165-178.

3 Southern KW, Munck A, Pollitt R, et al. A survey of newborn screening for cystic fibrosis in Europe. J Cyst Fibros 2007; 6: 57-65.

4 Castellani C, Picci L, Tamanini A, et al. Association between carrier screening and incidence of cystic fibrosis. JAMA 2009; 302: 2573-2579.

5 Watson MS, Cutting GR, Desnick RJ, et al. Cystic fibrosis population carrier screening: 2004 revision of American College of Medical Genetics mutation panel. Genet Med. 2004; 6: 387-391.

6 American College of Obstetricians and Gynecologists Committee on Genetics. ACOG Committee Opinion No. 486: update on carrier screening for cystic fibrosis. Obstet Gynecol. 2011; 117: 1028-1031.

7 Strom CM, Crossley B, Buller-Buerkle A, et al. Cystic fibrosis testing 8 years on: lessons learned from carrier screening and sequencing analysis. Genet Med 2011; 13: 166-172.

8 Miedzybrodzka ZH, Hall MH, Mollison J, et al. Antenatal screening for carriers of cystic fibrosis: randomised trial of stepwise $v$ couple screening. BMJ 1995; 310: 353-357.

9 Livingstone J, Axton RA, Gilfillan A, et al. Antenatal screening for cystic fibrosis: a trial of the couple model. $B M J$ 1994; 308: 1459-1462.

10 Cunningham S, Marshall T. Influence of five years of antenatal screening on the paediatric cystic fibrosis population in one region. Arch Dis Child 1998; 78: 345-348.

11 Witt DWC, Goonewardena P, Louie E, et al. Cystic fibrosis prenatal screening of 103,600 individuals in an HMO: molecular/clinical outcomes and a dramatic reduction in CF incidence. American Society of Human Genetics. Proceedings of Annual Meeting 2008. www.ashg.org/2008meeting/abstracts/fulltext/f20205.htm Date last accessed: October 29, 2013.

12 Cystic Fibrosis Foundation, Borowitz D, Parad RB, et al. Cystic Fibrosis Foundation practice guidelines for the management of infants with cystic fibrosis transmembrane conductance regulator-related metabolic syndrome during the first two years of life and beyond. J Pediatr 2009; 155: Suppl. 6, S106-S116.

13 Ren CL, Desai H, Platt M, et al. Clinical outcomes in infants with cystic fibrosis transmembrane conductance regulator (CFTR) related metabolic syndrome. Pediatr Pulmonol 2011; 46: 1079-1084.

14 Massie J, Curnow L, Gaffney L, et al. Declining prevalence of cystic fibrosis since the introduction of newborn screening. Arch Dis Child 2010; 95: 531-533.

15 Castellani C, Picci L, Scarpa M, et al. Cystic fibrosis carriers have higher neonatal immunoreactive trypsinogen values than non-carriers. Am J Med Genet A 2005; 135: 142-144.

16 Marteau T. Active cascade screening for cystic fibrosis. BMJ 1994; 308: 1468.

17 Sims EJ, Mugford M, Clark A, et al. Economic implications of newborn screening for cystic fibrosis: a cost of illness retrospective cohort study. Lancet 2007; 369: 1187-1195.

18 Norman R, van Gool K, Hall J, et al. Cost-effectiveness of carrier screening for cystic fibrosis in Australia. J Cyst Fibros 2012; 11: 281-287.

19 Radhakrishnan M, van Gool K, Hall J, et al. Economic evaluation of cystic fibrosis screening: a review of the literature. Health Policy 2008; 85: 133-147.

20 Kornreich R, Ekstein J, Edelmann L, et al. Premarital and prenatal screening for cystic fibrosis: experience in the Ashkenazi Jewish population. Genet Med 2004; 6: 415-420.

21 Stick SM. The first 2 years of life: implications of recent findings. Curr Opin Pulm Med 2009; 15: 615-620.

22 Ramsey BW, Davies J, McElvaney NG, et al. A CFTR potentiator in patients with cystic fibrosis and the G551D mutation. N Engl J Med 2011; 365: 1663-1672.

23 Study of ivacaftor in cystic fibrosis subjects 2 through 5 years of age with a CTFR gating mutation NCT01705145. http://clinicaltrials.gov/ct2/show/NCT01705145?term=ivacaftor\&rank=4 Date last accessed: October 31, 2013. Date last updated: September 12, 2013. 